\title{
Mechanism of action of posttransplantation cyclophosphamide: more than meets the eye
}

\author{
Vedran Radojcic' and Leo Luznik² \\ 'Division of Hematology and Hematologic Malignancies, University of Utah, Salt Lake City, Utah, USA. ²Division of Hematologic Malignancies, Johns Hopkins Sidney Kimmel Comprehensive Cancer Center, \\ Baltimore, Maryland, USA.
}

\begin{abstract}
For high-risk and refractory hematological malignancies, allogeneic hematopoietic stem cell transplantation (alloHSCT) is the only available curative therapy, with benefits derived from the antigenic disparity between recipient cancer and the incoming immune system. This immunologic mismatch can also lead to lethal graft-versus-host disease (CVHD), and immunosuppression strategies, including high-dose posttransplantation cyclophosphamide (PTCy), have been developed to allow for safe alloHSCT delivery. In this issue of $J C I$, Wachsmuth et al. present the results of preclinical studies designed to evaluate the mechanisms that underlie efficacy of PTCy after alloHSCT. The results of this study challenge previous reports indicating that alloreactive $\mathrm{T}$ cell elimination and thymic clonal deletion are primary mediators of PTCy efficacy and provide strong evidence to support FoxP3 ${ }^{+} \mathrm{CD4}^{+}$Tregs as important effectors of PTCy benefits.
\end{abstract}

\section{PTCy improves allogeneic bone} marrow transplant outcomes

Allogeneic hematopoietic stem cell transplantation (alloHSCT) remains the most effective curative therapy for patients with high-risk hematologic malignancies. Unfortunately, the development of acute and chronic graft-versus-host disease (GVHD) continues to affect the majority of transplant patients, causing high morbidity and mortality and consequent therapy failure. Administration of high-dose posttransplantation cyclophosphamide (PTCy) following alloHSCT has dramatically changed the therapeutic landscape of alloHSCT. PTCy has allowed almost universal access to the alloHSCT procedure through haploidentical donor use without a parallel increase in GVHD incidence or other immunosuppression-related toxicities $(1,2)$. Moreover, regardless of the degree of HLA matching or stem cell source for alloHSCT, PTCy effectively ablates chronic GVHD (1-5), thus enabling permanent discontinuation of immunosuppression in the majority of patients within the first year after transplantation $(2,6)$. In addition to being protected against GVHD, PTCy recipients maintain antiinfectious and antitumor responses (6-9), thus not compromising survival outcomes (10-12).

Despite clinical advances with the use of PTCy to improve alloHSCT outcomes, the mechanistic underpinnings of PTCy efficacy are not fully understood. Until recently, insight into PTCy action has been primarily driven by decades-old data obtained from experiments in MHCmatched murine models of skin allograft rejection $(13,14)$, in which selective elimination of alloantigen-reactive $\mathrm{CD} 4^{+}$but not $\mathrm{CD}^{+} \mathrm{T}$ cells was critical for preventing rejection (15). These highly contex-

Related Article: p. 2357

Conflict of interest: LL receives research support from Genentech and Merck and has a patent licensed to WindMIL Therapeutics (W02014085437A2)

Copyright: () 2019, American Society for Clinical Investigation.

Reference information: J Clin Invest. 2019;129(6):2189-2191. https://doi.org/10.1172/JCI128710.

tual observations have not been tested in alloHSCT models, in which more complex immune interactions are at play, and Tregs have recently been described as essential for the GVHD-protective benefits of PTCy $(16,17)$. Now, in this issue, Wachsmuth et al. demonstrate that previously proposed preferential elimination and clonal deletion of alloreactive $\mathrm{T}$ cells following cyclophosphamide use $(13,14)$ are not the dominant mechanisms needed for the beneficial effects of PTCy after alloHSCT, while solidifying the evidence supporting Treg importance in mediating long-term posttransplant tolerance and GVHD control with PTCy (18).

\section{Tregs are essential}

Wachsmuth et al. performed meticulously executed studies in multiple models of murine transplantation, with a particular focus on alloantigen-specific responses following PTCy to reach their conclusions. However, some limitations to the study remain. Authors base their findings mostly on a model of haploidentical transplantation $(\mathrm{B} 6 \mathrm{C} 3 \mathrm{~F} 1 \rightarrow \mathrm{B} 6 \mathrm{D} 2 \mathrm{~F} 1)$ in which the pathogenic role of $\mathrm{CD}^{+}$versus $\mathrm{CD} 8^{+}$ $\mathrm{T}$ cells remains undefined, thus limiting complete interpretation of study results. This is particularly relevant when superantigen responses are considered, as $\mathrm{CD}^{+}$ $\mathrm{T}$ cell responses are of questionable significance in mice and more so in humans. Nevertheless, experiments investigating the fate of transgenic $2 \mathrm{C}$ and $4 \mathrm{C} \mathrm{T}$ cells unequivocally demonstrated the limited impact PTCy has on alloreactive $\mathrm{T}$ cell proliferation, expansion, and persistence, despite parallel GVHD protection (though the latter was documented only for the $2 \mathrm{C}$ $\mathrm{T}$ cells). Wachsmuth et al. did not observe any measurable effects of PTCy on the posttransplant profile or recovery kinetics of $2 \mathrm{C} \mathrm{CD} 8^{+} \mathrm{T}$ cells, while only proliferation of $4 \mathrm{C} \mathrm{CD} 4^{+} \mathrm{T}$ cells was affected by the drug. The similarities between this work and the original studies of cyclophospha- 
mide and allograft tolerance end here, as the study by Wachsmuth et al. unquestionably dispels the notion that thymic function is necessary for PTCy-mediated GVHD protection and subsequently reinforces previous work to establish a role for Tregs in mediating PTCy benefits $(16,17)$. While the thymic independence of PTCy was hinted at in previous work describing the impact of distinct Treg subsets on PTCy outcome (16) and based on the observation that clinical PTCy benefits are maintained in adult human patients with limited thymic function, the current study by Wachsmuth and colleagues provides the definitive experimental proof. Several aspects regarding the role of Tregs in PTCy-mediated tolerance still require additional clarification, particularly regarding the duration of Treg benefit mediated by PTCy. Experiments in which Treg depletion can be induced validate the requirement for Tregs in mediating longterm alloHSCT tolerance; however, the precise impact of PTCy on the observed GVHD-like disease remains unclear. Notably, a GVHD-like condition that was distinct from the model-specific GVHD phenotype was also seen with Treg depletion following murine alloHSCT when GVHD was prevented without PTCy use (19).

\section{Conclusions and future directions}

The observations reported by Wachsmuth et al. lay particularly strong foundations for future studies, as their work provides decisive evidence that PTCy-dependent benefits are not mediated via alloreactive $\mathrm{T}$ cell elimination, while solidifying the evidence of Treg importance in delivering the PTCy outcome. Indeed, the sparing of the alloantigen-specific $\mathrm{T}$ cells and an overall negligible impact on $\mathrm{T}$ cell proliferation and expansion is the likely foundation behind the robust immune reconstitution and limited impact of PTCy on antiinfectious and antitumor responses. Moreover, the documented ability of PTCy-exposed $\mathrm{T}$ cells to respond to alloantigen further hints that immune responses remain preserved, though dedicated in vivo or in vitro experiments are needed to confirm this finding and to investigate its relevance for graft-versus-tumor responses.

Several of the observations made by Wachsmuth et al. will require careful attention while providing leads for future studies. For example, a clear difference between $\mathrm{CD}^{+}$and $\mathrm{CD} 4^{+} \mathrm{T}$ cell responses with PTCy use after allotransplantation in both hematopoietic cell and solid organ transplants - has now been repeatedly observed; however, the significance of this finding has not been fully queried. It is plausible that PTCy has distinct effects on T cell subsets and can thereby differentially mediate their functions after transplantation (e.g., antitumor or antiinfectious immunity vs. GVHD). Therefore, identification of these individual $\mathrm{T}$ cell roles could open new approaches to modulating posttransplant alloreactivity and optimizing PTCy-based GVHD prevention as a platform for cellular therapies after alloHSCT. These observations are highlighted in data demonstrating the impaired proliferation of PTCy-treated $\mathrm{CD}^{+}$and $\mathrm{CD}^{+}{ }^{+} \mathrm{T}$ cells in a mixed-lymphocyte reaction assay and the ability to transfer PTCy-mediated GVHD protection in the absence of Tregs (see Figures 6 and 7 in ref. 18), suggesting that $\mathrm{T}$ cell-intrinsic effects, which have thus far been largely ignored, may be essential for the long-term benefits of PTCy administration. In addition, the results of the Wachsmuth et al. study provide further guidance about when to query post-PTCy immune responses, as tolerizing imprinting occurs within a very short window after cyclophosphamide administration. These results provide clear fodder for dedicated investigation and further narrow down the time line within which PTCy modulates the alloresponse. Early control of the alloresponse is increasingly proving to be critical for long-term success of immunosuppression strategies, yet no other approaches are further reaching than PTCy. The robust chronic GVHD control, which is not seen with other novel acute GVHD-controlling strategies, such as sirolimus or IL-6 receptor inhibition (20-22), coupled with the ability to break the tolerance barriers, ease of use, and unequivocal evidence of outcomes comparable to standard alloBMT approaches, all provide for an enhanced access to transplantation across the globe. These clinical developments are made possible by the years of work aimed at increasing the understanding of how PTCy works, and now the study of Wachsmuth provides critical information for further advances.

\section{Acknowledgments}

Vedran Radojcic is supported by the Career Development Award from the American Society for Blood and Marrow Transplantation and the Huntsman Cancer Foundation.

Address correspondence to: Vedran Radojcic, University of Utah Huntsman Cancer Institute, Room 5263, 2000 Cir of Hope Drive, Salt Lake City, Utah 84105, USA. Phone: 801.213.6109; Email: vedran.radojcic@hsc.utah.edu. Or to: Leo Luznik, Johns Hopkins University, Cancer Research Building I, Room 2M88, 1650 Orleans Street, Baltimore, Maryland 21231, USA. Phone: 410.502.7732; Email: luznile@jhmi.edu.

1. Luznik L, et al. HLA-haploidentical bone marrow transplantation for hematologic malignancies using nonmyeloablative conditioning and highdose, posttransplantation cyclophosphamide. Biol Blood Marrow Transplant. 2008;14(6):641-650.

2. McCurdy SR, et al. Grade II acute graft-versushost disease and higher nucleated cell graft dose improve progression-free survival after HLA-haploidentical transplant with post-transplant cyclophosphamide. Biol Blood Marrow Transplant. 2018;24(2):343-352.

3. Luznik L, et al. High-dose cyclophosphamide as single-agent, short-course prophylaxis of graft-versus-host disease. Blood. 2010;115(16):3224-3230.

4. Kanakry CG, et al. Single-agent GVHD prophylaxis with posttransplantation cyclophosphamide after myeloablative, HLA-matched BMT for AML, ALL, and MDS. Blood. 2014;124(25):3817-3827.

5. Mielcarek M, et al. Posttransplantation cyclophosphamide for prevention of graft-versus-host disease after HLA-matched mobilized blood cell transplantation. Blood. 2016;127(11):1502-1508.

6. Kanakry CG, et al. Low immunosuppressive burden after HLA-matched related or unrelated BMT using posttransplantation cyclophosphamide. Blood. 2017;129(10):1389-1393.

7. Kanakry JA, et al. Absence of post-transplantation lymphoproliferative disorder after allogeneic blood or marrow transplantation using post-transplantation cyclophosphamide as graft-versus-host disease prophylaxis. Biol Blood Marrow Transplant. 2013;19(10):1514-1517.

8. Crocchiolo R, et al. Infections after T-replete haploidentical transplantation and high-dose cyclophosphamide as graft-versus-host disease prophylaxis. Transpl Infect Dis. 2015;17(2):242-249.

9. Tischer J, et al. Virus infection in HLA-haploidentical hematopoietic stem cell transplantation: incidence in the context of immune recovery in two different transplantation settings. Ann Hematol. 2015;94(10):1677-1688.

10. Ciurea SO, et al. Haploidentical transplant with posttransplant cyclophosphamide vs matched 
unrelated donor transplant for acute myeloid leukemia. Blood. 2015;126(8):1033-1040.

11. Kanate AS, et al. Reduced-intensity transplantation for lymphomas using haploidentical related donors vs HLA-matched unrelated donors. Blood. 2016;127(7):938-947.

12. Ghosh N, et al. Reduced-intensity transplantation for lymphomas using haploidentical related donors versus HLA-matched sibling donors: a center for international blood and marrow transplant research analysis. JClin Oncol. 2016;34(26):3141-3149.

13. Mayumi H, Good RA. Long-lasting skin allograft tolerance in adult mice induced across fully allogeneic (multimajor $\mathrm{H}-2$ plus multiminor histocompatibility) antigen barriers by a toleranceinducing method using cyclophosphamide. J Exp Med.1989;169(1):213-238.

14. Mayumi H, Umesue M, Nomoto K. Cyclophosphamide-induced immunological tolerance: an overview. Immunobiology. 1996;195(2):129-139.

15. Eto M, Mayumi H, Tomita Y, Yoshikai Y,

Nishimura Y, Nomoto K. Sequential mechanisms of cyclophosphamide-induced skin allograft tolerance including the intrathymic clonal deletion followed by late breakdown of the clonal deletion. J Immunol. 1990;145(5):1303-1310.

16. Ganguly S, et al. Donor CD4+ Foxp3+ regulatory $\mathrm{T}$ cells are necessary for posttransplantation cyclophosphamide-mediated protection against GVHD in mice. Blood. 2014;124(13):2131-2141.

17. Kanakry CG, et al. Aldehyde dehydrogenase expression drives human regulatory $\mathrm{T}$ cell resistance to posttransplantation cyclophosphamide. Sci Transl Med.2013;5(211):211ra157.

18. Wachsmuth LP, Patterson MT, Eckhaus MA, Venzon DJ, Gress RE, Kanakry CG. Posttransplantation cyclophosphamide prevents graftversus-host disease by inducing alloreactive $\mathrm{T}$ cell dysfunction and suppression. JClin Invest.
2019;129(6):2357-2373.

19. Radojcic $\mathrm{V}$, et al. STAT3 signaling in CD4+ T cells is critical for the pathogenesis of chronic sclerodermatous graft-versus-host disease in a murine model. JImmunol. 2010;184(2):764-774.

20. Kennedy GA, et al. Addition of interleukin-6 inhibition with tocilizumab to standard graftversus-host disease prophylaxis after allogeneic stem-cell transplantation: a phase $1 / 2$ trial. Lancet Oncol. 2014;15(13):1451-1459.

21. Drobyski WR, et al. Tocilizumab, tacrolimus and methotrexate for the prevention of acute graft-versus-host disease: low incidence of lower gastrointestinal tract disease. Haematologica. 2018;103(4):717-727.

22. Kornblit B, et al. A randomized phase II trial of tacrolimus, mycophenolate mofetil and sirolimus after non-myeloablative unrelated donor transplantation. Haematologica. 2014;99(10):1624-1631. 